\title{
High current hollow cathode as a radiometric transfer standard source for the extreme vacuum ultraviolet
}

\author{
Karsten Danzmann, Mathias Günther, Joachim Fischer, Manfred Kock, and Michael Kühne
}

\begin{abstract}
A high current hollow cathode source has been developed as a radiometric source standard for the extreme VUV. The source is operated at a constant current of $2 \mathrm{~A}$ with an aluminum cathode and helium or argon as buffer gas at pressures of $\sim 1 \mathrm{hPa}$. The radiance of spectral lines in the wavelength range from 13 to $60 \mathrm{~nm}$ has been determined by comparison to the calculable spectral radiant power of the electron storage ring BESSY. An uncertainty of the order of $13 \%$ has been achieved for most of the investigated lines. The spectral emission is stable to better than $5 \%$ for an operating time of $30 \mathrm{~h}$. An exchange of the cathode restores the original radiance.
\end{abstract}

\section{Introduction}

Over the last few years the interest in VUV spectroscopy has been steadily growing. This holds especially for fields like basic research, controlled nuclear fusion (both magnetic and inertial confinement), and spacebased astronomy. In all these areas quantitative spectroscopy is of great importance requiring the availability of radiometric standards. In principle, quantitative spectroscopy can be based on either source standards or detector standards, but in the latter case the transmittance of the optical imaging system and the monochromator must be known. ${ }^{1}$ As the determination of the transmittance can be complicated and especially in the extreme VUV the transmittance of optical systems can change dramatically in time due to contamination and other aging effects, it is often preferrable to work with radiometric source standards.

While the vacuum ultraviolet (VUV) extends from $\sim 200 \mathrm{~nm}$ (cutoff due to atmospheric absorption) to $\sim 0.2 \mathrm{~nm}$ (onset of transparency), it is often useful to subdivide this range into a long-wavelength VUV from 200 to $105 \mathrm{~nm}$ and an extreme VUV region below 105 $\mathrm{nm}$, as below this wavelength no solid window materi-

\footnotetext{
When this work was done K. Danzmann, J. Fischer, and M. Kühne were with PTB-Berlin Institute, Abbestrasse 2-12, D-1000 Berlin 10, Federal Republic of Germany; K. Danzmann is now with Stanford University, Physics Department, Stanford, California 94305. The other authors are with University of Hanover, Institute for Plasmaphysics, D-3000 Hanover 1, Federal Republic of Germany.

Received 18 March 1988.

$0003-6935 / 88 / 234947-05 \$ 02.00 / 0$.

(C) 1988 Optical Society of America.
}

als exist, so that different experimental techniques are required. The wavelength region below $30 \mathrm{~nm}$ is often also called the soft x-ray region. For the long-wavelength VUV radiometric source standards like the wall-stabilized $\operatorname{arcs}^{2-4}$ and deuterium lamps $s^{5-7}$ are available. Wall stabilized arcs with differential pumping systems offer in principle the possibility of extending the useful range into the $\mathrm{XUV}^{4,8}$ but are limited by the onset of the helium resonance continuum to wavelengths longer than $53 \mathrm{~nm}$. Operating at high pressures they require large and costly differential pumping systems, and the only system operated down to $53 \mathrm{~nm}$, the hydrogen arc, needs a lot of expertise to operate.

If bright continuum sources are required the only solution in this wavelength range is a short-duration pulsed source that can operate in vacuum conditions. Laser produced plasmas with pulse durations in the nanosecond regime have been shown to be suitable for transfer source standard application. ${ }^{9}$

For many purposes, however, line emission sources are better suited than continuum sources. Such sources can be operated with comparatively low gas pressures requiring, therefore, only modest differential pumping systems and do not suffer seriously from self-absorption if a properly designed differential pumping system is used.

In this paper a high current hollow cathode source is described that can serve as a radiometric transfer source standard. The design and calibration of the source and its radiometric properties are discussed.

\section{Hollow Cathode Source}

\section{A. Discharge Configuration}

Based on earlier versions ${ }^{10,11}$ the hollow cathode (HC) was jointly developed at the Institut für Plasma- 
physik in Hannover and the PTB in Berlin for use as a line-radiation standard in the VUV.

A longitudinal section of the rotational-symmetric source is shown in Fig. 1. There are two anodes and one cathode, all made of stainless steel and watercooled. The cathode insert, a hollow cylinder with a central bore of $8 \mathrm{~mm}$ and a length of $60 \mathrm{~mm}$, is held in the cathode body by a slightly conical fitting. The conical shape of the anodes reduces both arcing and short circuiting through metal depositions due to sputtering from the probe cylinder. Central bores of $4 \mathrm{~mm}$ in both anodes allow end-on observation of the plasma.

The electrodes are adjusted and electrically insulated by precisely manufactured glass spacers, which are damage protected by Delrin caps. The whole system is vacuum sealed by Viton O-rings.

A two-stage differential pumping system enables windowless observation in the VUV. The flux limiting aperture $A$ (see Fig. 1) with a diameter of $1.2 \mathrm{~mm}$ limits the solid angle for the observable radiation to $3 \times 10^{-4}$ sr. To avoid clogging of the aperture, the gas inlet is located between the anode and the first pumping stage with a bore of $1.5 \mathrm{~mm}$. The $\mathrm{HC}$ is supported by a fork mount with the pivot in the aperture avoiding a shift of the aperture during alignment.

The HC is operated with a current-stabilized power supply $(3 \mathrm{kV}, 3 \mathrm{~A})$. The anodes are maintained at ground potential, whereas the cathode is at high negative voltage. A $50-\Omega$ ballast resistor is employed to stabilize the discharge and to suppress short circuits. The buffer gas used is argon (purity $99.9993 \%$ ) or helium (purity 99.9996\%) at a pressure of the order of 1 $\mathrm{hPa}$. The cathode inset is aluminum with a $99.5 \%$ degree of purity. By means of a throttle valve the gas inflow is adjusted to compensate the outflow through the differential pumping system. At a pressure of 1 $\mathrm{hPa}$ the typical flow rate is of the order of 1 bar $1 / \mathrm{h}$. At a current of $2 \mathrm{~A}$ the discharge burns reproducibly (changes in spectral line emission, $\leq 5 \%$ ) for more than $30 \mathrm{~h}$. After cleaning the anodes and the glass spacers with diluted hydrochloric acid and replacing the cathode inset, the $\mathrm{HC}$ is ready again for operation with the original emission values restored.

\section{B. Discharge Parameters and Operation Characteristics}

The spectral emission of the $\mathrm{HC}$ depends basically on the discharge current and voltage drop across the electrodes. The $\mathrm{HC}$ has always been operated with a fixed current, adjusting the voltage drop by regulating the buffer gas pressure. The radiance of the spectral lines depends approximately linearly on the current in the case of the noble gas lines and approximately quadratically in the case of metal ions sputtered off the hollow cathode. ${ }^{11}$ Because of this current dependence the stabilization of the discharge current has to be better than $1 \%$.

The correlation between discharge voltage and buffer gas pressure can be seen in Fig. 2. The sensitivity of the voltage allows precise adjustment of the buffer gas pressure. For example, in an $\mathrm{Al}-\mathrm{He}$ discharge at a current of $2 \mathrm{~A}$ a voltage of $400 \mathrm{~V}$ is reached at a pressure

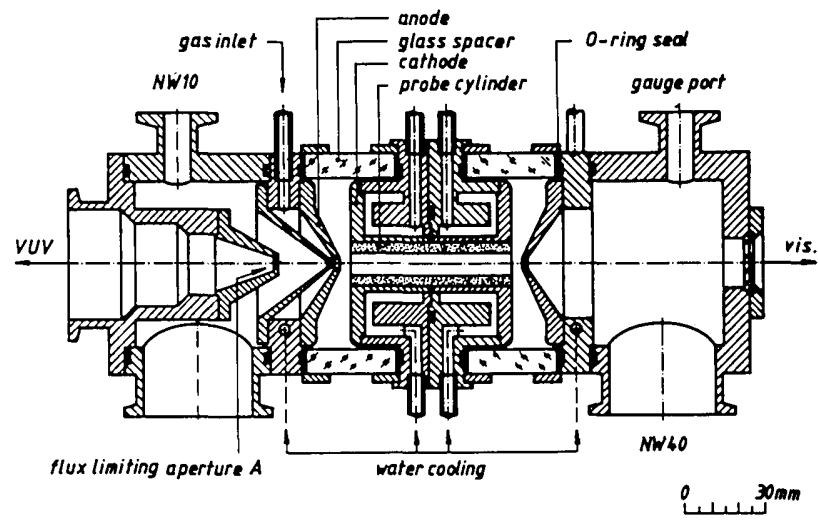

Fig. 1. Longitudinal section of the hollow cathode with integrated two-stage differential pumping system.

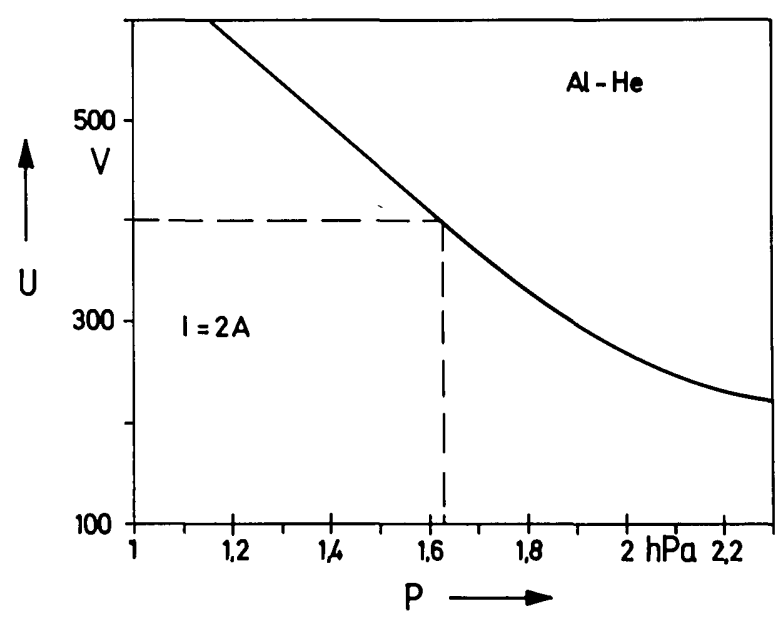

Fig. 2. Discharge voltage as a function of buffer gas pressure. The emission of the $\mathrm{HC}$ is calibrated at the working point $(2 \mathrm{~A}, 400 \mathrm{~V})$.

of $1.64 \mathrm{hPa}$. This working point $(2 \mathrm{~A}, 400 \mathrm{~V})$ was chosen for all investigations, independent of the met$\mathrm{al} / \mathrm{rare}$ gas combination used.

The radial homogeneity across the flux limiting aperture and the isotropy of the radiation emission were investigated for different spectral lines. For that purpose, the aperture $A$ was imaged with a spherical mirror onto the entrance slit of an 1-m normal-incidence VUV monochromator (McPherson). A concave grating with 1200 lines/mm gave a reciprocal linear dispersion of $0.83 \mathrm{~nm} / \mathrm{mm}$ in the first order. The VUV signals were detected by an EMI $9605 / 3 \mathrm{~B}$ electron multiplier as well as a sodium salycilate-coated EMI D299/612 multiplier, which were operated in conjunction with a photon counting system (EG\&G/Ortec).

The angular distribution of the radiation in a single spectral line was measured within a solid angle of $6 \times$ $10^{-7} \mathrm{sr}$ defined by the imaging aperture in front of the spherical mirror. The entrance slit of the monochromator was opened to $1.4 \mathrm{~mm}$ so that the full image of the $\mathrm{HC}$ aperture could be used. The angular distribution of the radiation emission was obtained by pivoting the source around an axis through aperture $A$ perpendicular to the direction of observation. In the investi- 
gated angular range of $\pm 16 \mathrm{mrad}$ the angular distribution can be approximated by a parabola (Fig. 3). For values of $\leq 10^{-4} \mathrm{sr}$, as indicated by the shaded area in Fig. 3, the emission can be considered quasi-isotropic.

To determine the radial distribution of the radiation emission across the aperture, a horizontal slit of 150 $\mu \mathrm{m}$ width was placed symmetrically in front of aperture $A$. The entrance slit of the monochromator was closed to $50 \mu \mathrm{m}$ so that a rectangular area of $50 \times 150$ $\mu \mathrm{m}$ of the hollow cathode source was viewed. The hollow cathode source was then moved perpendicular to the axis of observation, and the corresponding radial emission profile was recorded (Fig. 4). It can be seen that the profile is essentially flat across the $1.2-\mathrm{mm}$ aperture.

\section{Radiance of Spectral Lines}

The radiances of the emitted spectral emission lines were determined in the VUV-radiometric laboratory of PTB at the Berlin electron storage ring BESSY. As BESSY can be used as a primary standard of spectral irradiance in the VUV and soft $\mathrm{x}$-ray region, ${ }^{12,13}$ the radiances of the spectral lines emitted by the hollow cathode source were determined by radiant flux comparison. An ellipsoidal mirror images the tangent point of the storage ring or the hollow cathode source into a toroidal grating monochromator so that the radiant flux is not limited by the entrance slit size (Fig. 5). For both sources the same surface element of the mirror, the same angle of incidence $\left(86^{\circ}\right)$, and a common aperture stop $B$ are used. To allow for corrections due to the different degree of polarization of the two sources (synchrotron radiation is highly polarized near the electron orbit plane), the monochromator can be rotated $90^{\circ}$ around its optical axis defined by the center of the entrance slit and the center of the grating.

The distance from the storage ring tangent point to the mirror was $15,000 \mathrm{~mm}$, and the distance from the 1.2-mm aperture $A$ in the differential pumping system of the hollow cathode to the mirror was $5370 \mathrm{~mm}$. The corresponding images were formed in a distance of 930 and $1045 \mathrm{~mm}$, respectively. The distance between the monochromator and the mirror could be adjusted to compensate for the different image distances. Gratings with 200 and 600 lines $/ \mathrm{mm}$ were used with corresponding exit slit widths of 50 and $150 \mu \mathrm{m}$, respectively, to ensure a bandwidth of $<0.1 \mathrm{~nm}$ for all wavelengths. The VUV signal was detected by a fast linear focused EMI D233B electron multiplier.

The radiant flux $\phi^{\mathrm{HC}}$ integrated over the line profile is given by

$$
\phi^{\mathrm{HC}}=\int \phi_{\lambda}^{\mathrm{HC}}(\lambda) d \lambda=\int \phi_{\lambda}^{\mathrm{SR}}(\lambda) F(\lambda) i^{\mathrm{HC}}(\lambda) / i^{\mathrm{SR}}(\lambda) d \lambda,
$$

where $\phi_{\lambda}^{\mathrm{SR}}(\lambda)$ is the calculable spectral radiant flux of the synchrotron radiation according to Schwinger ${ }^{14}$ and where $F(\lambda)$ describes the factor due to the different degrees of polarization of the two sources (for details, see 15). $i^{\mathrm{HC}}(\lambda)$ and $i^{\mathrm{SR}}(\lambda)$ are the spectral photocurrents of the two sources.

The radiant flux integrated over the line profile is then converted into radiant intensity by dividing with

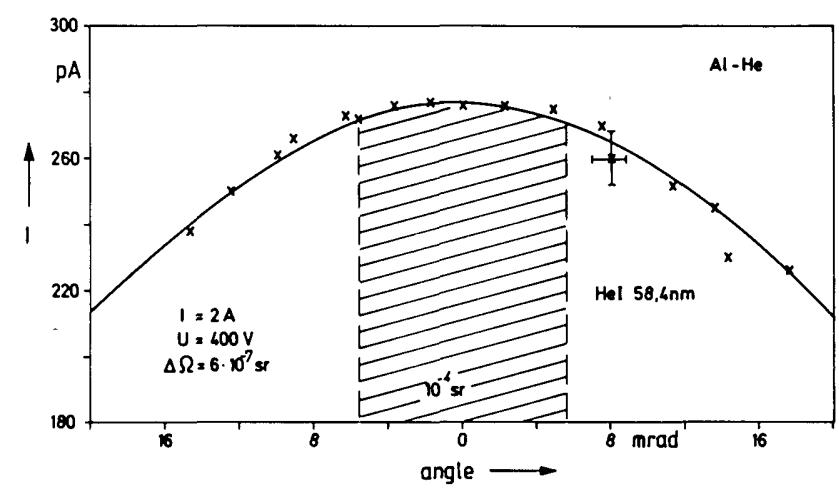

Fig. 3. Angular characteristic of radiation emission. To obtain the characteristic the hollow cathode was pivoted around an axis passing through the flux-limiting aperture $A$ perpendicular to the direction of observation. The angular resolution was $6 \times 10^{-7} \mathrm{sr}$.

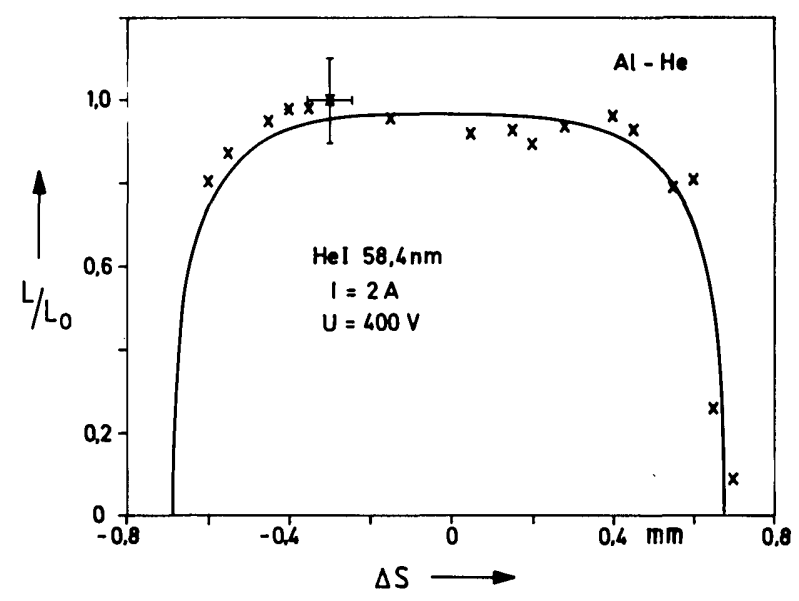

Fig. 4. Irradiance distribution over the cross section of the measuring aperture. A section of $50 \times 150 \mu \mathrm{m}^{2}$ was used to obtain a single measuring point.

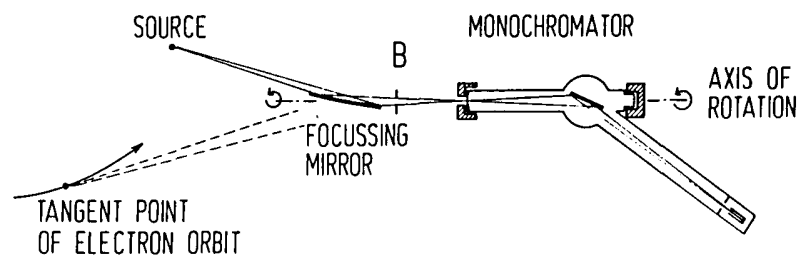

Fig. 5. Instrumentation for comparing at grazing incidence the unknown spectral radiant flux of the hollow cathode source with the calculated spectral radiant flux of the electron storage ring BESSY.

the solid angle $\Omega$ during the hollow cathode measurement and into radiance by additionally averaging over the source size (aperture with 1.2-mm diameter and area $A$ ):

$$
I^{\mathrm{HC}}=\phi^{\mathrm{HC}} / \Omega \quad L^{\mathrm{HC}}=\phi^{\mathrm{HC}} /(\Omega A) .
$$

The solid angle $\Omega=2.27 \times 10^{-7} \mathrm{sr}$ is given by the effective size of the aperture stop $B(2.56 \times 2.56 \mathrm{~mm})$ in the distance $5370 \mathrm{~mm}$ from the source and is much smaller than the maximum angle of $1 \times 10^{-4} \mathrm{sr}$ for flat angular distribution mentioned above. Using source 
sizes other than $1.2 \mathrm{~mm}$, the radiances quoted have to be converted according to Fig. 4 .

Measuring both the He II Lyman series lines and the synchrotron radiation at the corresponding wavelengths, an aluminum filter of $0.2-\mu \mathrm{m}$ thickness was used to avoid higher order corrections due to the monochromator grating. The radiance determination of these lines with the 200 -line $/ \mathrm{mm}$ grating and the 600 line/mm grating yielded to the same results which confirmed a sufficient higher order suppression by the filter. To measure the $\mathrm{He}$ I line at $58.4 \mathrm{~nm}$ a SnGe filter ( $\mathrm{Sn}$ with $3 \% \mathrm{Ge}$; thickness, $0.16 \mu \mathrm{m}$ ) was employed. In these conditions and under the operating parameters mentioned above, with helium as buffer gas the $\mathrm{Al}$ IV doublet at $16 \mathrm{~nm}$, the He II Lyman series and the $\mathrm{He} \mathrm{I}$ lines were measured. If argon is used an additional $\mathrm{Al} \mathrm{IV}$ line appears at $13 \mathrm{~nm}$ (Table I). The emitted radiances are stable to within $5 \%$ for an operating period of $30 \mathrm{~h}$. A second hollow cathode with the same design showed radiances which differed from the results of the first one by no more than the uncertainties quoted in the last column of Table $I$.

\section{A. Estimation of Uncertainties}

According to Eqs. (1) and (2) the systematic uncertainty squared of $L^{\mathrm{HC}}$ is determined by

Table I. Radlance of Selected Spectral Lines of the Hollow Cathode $I=2$ $A, U=400 \mathrm{~V}$

\begin{tabular}{lccccc}
\hline & & & & \multicolumn{3}{c}{ Uncertainty } \\
$\lambda(\mathrm{nm})$ & Ion & Buffer gas & $I(\mathrm{~W} / \mathrm{sr})$ & $L\left(\mathrm{~W} / \mathrm{m}^{2} \mathrm{sr}\right)$ & $\%$ \\
\hline 13.1 & $\mathrm{Al} \mathrm{IV}$ & $\mathrm{Ar}$ & $1.5 \times 10^{-7}$ & 0.13 & 25 \\
$16.0 / 16.2$ & $\mathrm{Al} \mathrm{IV}$ & $\mathrm{Ar}$ & $1.91 \times 10^{-6}$ & 1.69 & 13 \\
$16.0 / 16.2$ & $\mathrm{Al} \mathrm{IV}$ & $\mathrm{He}$ & $1.53 \times 10^{-6}$ & 1.35 & 13 \\
24.3 & $\mathrm{He} \mathrm{II}$ & $\mathrm{He}$ & $4.65 \times 10^{-5}$ & 41.1 & 13 \\
25.6 & $\mathrm{He} \mathrm{II}$ & $\mathrm{He}$ & $1.47 \times 10^{-4}$ & 130 & 13 \\
30.4 & $\mathrm{He} \mathrm{II}$ & $\mathrm{He}$ & $6.76 \times 10^{-4}$ & 598 & 13 \\
58.4 & $\mathrm{He} \mathrm{I}$ & $\mathrm{He}$ & $1.53 \times 10^{-3}$ & 1350 & 13 \\
\hline
\end{tabular}

Table II. Systematic Relative Uncertainty Contributions

(a) Radiant flux of synchrotron radiation $\Delta \phi_{\lambda}^{\mathrm{SR}} / \phi_{\lambda}^{\mathrm{SR}}$

$1.6 \times 10^{-2}$

(b) Polarizing properties of the ellipsoidal mirror and the monochromatordetector system and degree of polarization of synchrotron radiation $\Delta F / F$

(c) Local efficiency variation of monochromator grating $\Delta \epsilon / \epsilon$

(d) Ratio of photocurrent $\Delta\left(i^{\mathrm{HC}} / i^{\mathrm{SR}}\right) /$ $\left(i^{\mathrm{HC}} / i^{\mathrm{SR}}\right)$

$5.4 \times 10^{-1}$

e) Solid angle of hollow cathode measurements $\Delta \Omega / \Omega$

(f) Area of aperture in the differential pumping system of the hollow cathode $\Delta A / A$

Overall systematic relative uncertainty (addition in quadrature)

$$
\begin{aligned}
\left\{\Delta L^{\mathrm{HC}} / L^{\mathrm{HC}}\right\}^{2}= & \left\{\Delta \phi_{\lambda}^{\mathrm{SR}} / \phi_{\lambda}^{\mathrm{SR}}\right\}^{2}+\{\Delta F / F\}^{2}+\{\Delta \epsilon / \epsilon\}^{2} \\
& +\left\{\Delta\left(i^{\mathrm{HC}} / i^{\mathrm{SR}}\right) /\left(i^{\mathrm{HC}} / i^{\mathrm{SR}}\right)\right\}^{2}+\{\Delta \Omega / \Omega\}^{2}+\{\Delta A / A\}^{2}
\end{aligned}
$$

in which $\Delta \epsilon / \epsilon$ accounts for the uncertainty caused by the local efficiency variation of the monochromator grating. The contributions to the systematic uncertainty are listed in Table II.

The uncertainty of the radiant flux of the synchrotron radiation is mainly due to the uncertainties of the measured storage ring parameters energy and current of the electrons and the magnetic induction at the tangent point of observation; details are in Ref. 13. For a comprehensive treatment of the uncertainties of $F$ (polarization), see Ref. 15. The uncertainty arising from local efficiency variations of the monochromator grating is caused by the different distances from the ellipsoidal mirror to the electron storage ring and the hollow cathode, respectively. By use of apertures of different sizes the uncertainty $\Delta \epsilon / \epsilon$ was determined to $\leq 5 \%$. The main contribution to the uncertainty of the ratio of the photocurrent $i \mathrm{HC} / i^{\mathrm{SR}}$ is caused by remaining higher diffraction orders of the monochromator grating. The uncertainties of the solid angle $\Omega$ and the radiating area $A$ arose from measurement of the dimensions of the corresponding apertures.

Addition in quadrature of the uncertainties according to Table II and of the standard deviations of the single radiance determinations led to the relation uncertainties quoted in the last column of Table I (2- $\sigma$ level).

\section{Summary}

A high current hollow cathode has been developed that can be used as a radiometric transfer standard of radiance in the extreme VUV. The source uses an aluminum cathode and argon or helium as buffer gas at pressures of $\sim 1 \mathrm{hPa}$ operating at a current of $2 \mathrm{~A}$. The radiances of spectral lines of both the cathode material and the buffer gas have been calibrated within the spectral range from 13 to $60 \mathrm{~nm}$ by comparison with the calculable spectral radiant power of the electron storage ring BESSY. An uncertainty of the order of $13 \%$ has been achieved for most lines. The spectral emission is stable within $5 \%$ for $30 \mathrm{~h}$ of operation time. The exchange of the cathode will restore the emission to its original value.

\section{References}

1. M. Kühne and B. Wende, "VUV and Soft X-Ray Radiometry," J. Phys. E 18, 637 (1985).

2. J. M. Bridges and W. R. Ott, "Vacuum Ultraviolet Radiometry. 3: The Argon Mini-arc as a New Secondary Standard of Spectral Radiance," Appl. Opt. 16, 367 (1977).

3. D. Einfeld, K. Grützmacher, and D. Stuck, "On the Use of a Low Current Argon Arc with a $\mathrm{MgF}_{2}$ Window as a VUV Transfer Standard of the Spectral Radiance (125 nm $\leq \lambda \leq 335 \mathrm{~nm})$," Z. Naturforsch. Teil A 34, 233 (1979).

4. R. C. Preston, C. Brookes, and F. W. J. Clutterbuck, "Vacuum Ultraviolet Radiance Transfer Standard Based on an Argon Miniarc with Integral Differential Pumping Unit," J. Phys. E 13, 1206 (1980). 
5. R. D. Saunders, W. R. Ott, and J. M. Bridges, "Spectral Irradiance Standard for the Ultraviolet: the Deuterium Lamp," Appl. Opt. 17, 593 (1978).

6. D. Einfeld, D. Stuck, and B. Wende, "Calibration of Radiometric Transfer Standards in the UV and VUV by Electron Synchrotron Radiation Using a Normal Incidence Radiometer," Metrologia 14, 111 (1978).

7. P. J. Key and R. C. Preston, "Magnesium Fluoride Windowed Deuterium Lamps as Radiance Transfer Standards Between $115 \mathrm{~nm}$ and $370 \mathrm{~nm}$," J. Phys. E 13, 866 (1980).

8. K. Behringer and P. Thoma, "VUV Radiometry Below $100 \mathrm{~nm}$ : the Highpower Hydrogen Arc as a Standard Source of Continuum Radiation Between $53 \mathrm{~nm}$ and $92 \mathrm{~nm}$," Appl. Opt. 18, 2586 (1979).

9. J. Fischer, M. Kühne, and B. Wende, "Laser-Produced Plasmas as Radiometric Transfer-Standard Sources for the VacuumUltraviolet and the Soft X-Ray Range," Metrologia 23, 179 (1986/1987).

10. K. Danzmann and M. Kock, "Oscillator Strengths of Ti II from
Combined Hook and Emission Measurements," J. Phys. B 13, 2051 (1980).

11. K. Danzmann, J. Fischer, and M. Kühne, "A High Current Hollow Cathode as a Source of Intense Line Radiation in the VUV," J. Phys. D 18, 1299 (1985).

12. F. Riehle and B. Wende, "Electron Storage Ring BESSY as a Radiometric Source of Calculable Spectral Radiant Power Between $0.5 \mathrm{~nm}$ and $1000 \mathrm{~nm}$," Opt. Lett. 10, 365 (1985).

13. F. Riehle and B. Wende, "Ein Elektronenspeicherring als primäres Strahlungsnormal zur Realisierung strahlungsoptischer Einheiten vom Infraroten bis in den Bereich weicher Röntgenstrahlung," Optik 75, 142 (1987).

14. J. Schwinger, "On the Classical Radiation of Accelerated Electrons," Phys. Rev. 75, 1912 (1949).

15. J. Fischer, M. Kühne, and B. Wende, "Spectral Radiant Power Measurements of VUV and Soft X-Ray Sources Using the Electron Storage Ring BESSY as a Radiometric Standard Source," Appl. Opt. 23, 4252 (1984).
NASA continued from page 4946

\section{Metal/ceramic bond coatings for high temperatures}

A new class of reduced-thermal-expansion bond coatings has been developed for use at high temperatures in thermal-barrier-coating systems. These bond coatings are composed of low-pressure-plasma-sprayed metallic matrices dispersed with low-thermal-expansion, high-bulk-modulus ceramic particles. The new coatings and the method of application represent improvements over the earlier practice of applying a ceramic coat directly over a metallic bond coat in that they lower the thermal-expansion-mismatch strain while maintaining integrity at high temperatures. The dispersoids are initially processed to possess high cohesive strength, low porosity, and generally spherical shapes. The anticipated optimum microstructure would be obtained by adjusting plasma-spraying conditions so that the metallic particles melt completely while the dispersoids exhibit only surface melting and remain roughly spherical.

The resulting bond coat ideally consists of a dense matrix of metal with well-bonded, crudely spherical ceramic dispersoids. However, in practice, many dispersoids may melt completely and flatten on impact. The volume fraction of the ceramic is kept sufficiently low so that the dispersoids tend not to be in contact with each other, thereby enabling the dispersoids to constrain the thermal expansion of the metal matrix while maintaining inherent resistance to oxidation.

This work was done by Robert A. Miller of Lewis Research Center and George W. Leissler of Sverdrup Technology, Inc. Refer to LEW-14541.

\section{Schottky diode with surface channel}

An improved configuration for a Schottky-barrier diode reduces the parasitic shunt capacitance. Schottky-barrier devices are used as microwave mixer and varactor diodes, and shunt capacitance degrades performance at the typical operating frequencies above 30 $\mathrm{GHz}$. The new configuration avoids the need for the special skill and equipment required to make contact-whisker Schottky-barrier diodes, which have low shunt capacitance. As in previous whiskerless designs, the contact whisker is replaced by an integrally fabricat- ed anode contact finger. However, the new configuration eliminates some of the difficulties encountered in the fabrication of prior morecomplicated whiskerless Schottky diodes of low shunt capacitance.

The parasitic shunt capacitance is reduced by removing a portion of the high-dielectric-permeability and conductive semiconductor material from a region adjacent to the anode contact finger. If not removed, this material would support conduction and displacement currents between the anode contact finger and the metal/semiconductor junction, thereby giving rise to a relatively high shunt capacitance. The fabrication process and the configuration of the diode are shown in Fig. 8. The first step is the prefabrication of an epitaxial wafer that consists of a semi-insulating GaAs substrate layer, an intermediate buffer layer of $n^{+} \mathrm{GaAs}$, and an upper layer of $n \mathrm{GaAs}$. An insulating layer, preferably of $\mathrm{SiO}_{2}$, is deposited on the upper $n$ GaAs layer.

Space for an ohmic contact pad is formed by the use of a photoresist pattern and etching through the $\mathrm{SiO}_{2}$ and, in some cases, the GaAs layers. The ohmic-contact metal is deposited by electroplating a sequence of tin, tin/nickel, and gold over the contact-pad area. The contact is alloyed, then overlaid with gold. The anode region is defined by a photoresist pattern, and the anode window is etched through the $\mathrm{SiO}_{2}$ layer. The photoresist is then removed, and a thin layer of platinum followed by a thicker layer of gold are plated into the anode window and onto the $n$ GaAs to form the anode. A thin layer of chromium and another of gold are then sputtered onto the entire upper surface. A photoresist is applied and patterned to define the anode contact pad and anode contact finger. A thick layer of gold for the pad and finger is then deposited. The photoresist is removed, and the thinner layers of gold and chromium are etched away, thus forming the anode contact pad and finger. A photoresist is then applied and patterned to define the region from which the material will be removed to reduce the shunt capacitance. After the etching of the $\mathrm{SiO}_{2}$ in this region, the photoresist is removed and the region is etched further, forming a channel that extends under the anode contact finger and into the semi-insulating GaAs layer.

This process allows for easy variation of the length, width, and thickness of the anode contact finger, thus enabling the design of the finger for optimum inductance. The size of the anode contact pad can also be varied easily for optimum coupling of power into the device. The depth and width of the channel can be adjusted to minimize shunt capacitance. Thus, design changes can be executed through routine processing variations to make devices that have parasitic series resistances and shunt capacitances approaching theoretical limits.

continued on page 4959 